hep-th/0510060

\title{
Counting chiral primaries in $\mathcal{N}=1 d=4$ superconformal field theories
}

\author{
Christian Römelsberger \\ Perimeter Institute \\ 31 Caroline St. N. \\ Waterloo, ON N2L 2Y5, Canada
}

\begin{abstract}
I derive a procedure to count chiral primary states in $\mathcal{N}=1$ superconformal field theories in four dimensions. The chiral primaries are counted by putting the $\mathcal{N}=1$ field theory on $S^{3} \times \mathbb{R}$. I also define an index that counts semi-short multiplets of the superconformal theory. I construct $\mathcal{N}=1$ supersymmetric Lagrangians on $S^{3} \times \mathbb{R}$ for theories which are believed to flow to a conformal fixed point in the IR. For ungauged theories I reduce the field theory to a supersymmetric quantum mechanics, whereas for gauge theories I use chiral ring arguments. I count chiral primaries for $S U(2)$ SYM with three flavors and its Seiberg dual. Those two results agree provided a new chiral ring relation holds.
\end{abstract}

October 2005 


\section{Contents}

1 Introduction $\quad 3$

2 Group Theorv 5

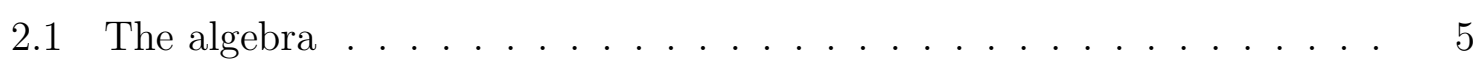

2.2 Chiral primaries . . . . . . . . . . . . . . . . . . . . . . 7

2.3 An inded . . . . . . . . . . . . . . . . . . . . 8

3 Killing Spinors 10

4 Construction of the Field Theorv 12

4.1 Chiral multiplets and the Wess-Zumino model . . . . . . . . . . . . 13

4.2 Vector multiplets . . . . . . . . . . . . . . . . . . . . . . 14

4.3 Gauge invariant interactions . . . . . . . . . . . . . . . . 16

4.4 A twist . . . . . . . . . . . . . . . . . . . 16

5 Ungauged theories $\quad 18$

5.1 Reduction to quantum mechanics . . . . . . . . . . . . . 18

5.2 The canonical formalism . . . . . . . . . . . . . . . . . . . . . . . . . . 19

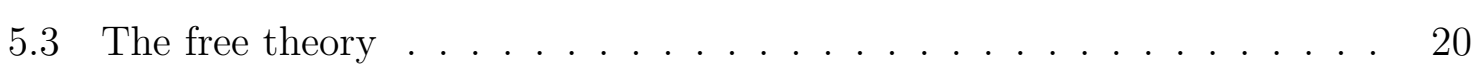

5.4 The Wess-Zumino model and the chiral ring . . . . . . . . . . . . . . . 21

6 Gauge theories and the chiral ring $\quad 23$

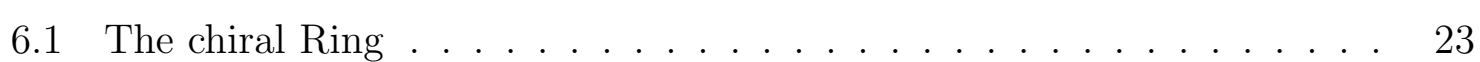

6.2 Seiberg dualitv for $S U(2)$ super Yang Mills theorv with 3 flavors . . . . 25

6.3 Seiberg duality in the conformal window . . . . . . . . . . . . . . . . . 27

$\begin{array}{ll}\text { A Some conventions } & 29\end{array}$

B Invariant forms and Killing vectors on $S U(2) \quad 30$ 


\section{Introduction}

Recently there has been a lot of progress in understanding the AdS-CFT correspondence 1, 2, 3, 4. A big part of this progress was made by restricting the gauge theory on $S^{3} \times \mathbb{R}$ to a BPS sector. In this BPS sector the gauge theory only the lowest harmonics of the fields contribute to the partition function and the gauge theory simplifies to matrix quantum mechanics.

In this paper I want to restrict my attention just to gauge theories on $S^{3} \times \mathbb{R}$ without looking at the gravity side. I am considering the chiral sector of $\mathcal{N}=1$ field theories. In flat space this sector is only partly described by quantum mechanics [5], actually it is not quantum mechanics, but a matrix integral. On $S^{3} \times \mathbb{R}$ there is one obvious correlation function that is calculated by doing a restriction to quantum mechanics. This is the index ${ }^{1}$

$$
\operatorname{ind}\left(e^{-\beta}\right)=\operatorname{tr}(-1)^{F} e^{-\frac{3 \beta}{2}\left(H-\frac{1}{2} J\right)} .
$$

This index counts semi-short multiplets of the superconformal algebra ${ }^{2}$. A similar index is the Witten index [8], which is calculated for supersymmetric field theories on $T^{3} \times \mathbb{R}$. The Witten index counts supersymmetric vacua in flat space as opposed to BPS states.

In this paper, however, I am trying to develop a machinery for counting chiral primaries, which are a subset of the semi-short multiplets. The chiral primaries saturate a second BPS bound. There doesn't seem to be an index counting those states.

It is in general hard to write down Lagrangians for $\mathcal{N}=1$ superconformal field theories. Typically those theories are defined through some field theory in the UV which is believed to flow to a superconformal fixed point in the IR. The matter content and the superpotential in the UV define the universality class of the theory. The chiral primaries of such theories are often written down in terms of gauge invariant chiral operators made out of the lowest components of the chiral superfields in the Lagrangian.

The index is a topological quantity and can be calculated in a weak coupling regime in the UV using the Born-Oppenheimer approximation 9]. Similarly one can use the

\footnotetext{
${ }^{1}$ In the course of this work related work has been published [6]. Part of the results of this work was presented in [7].

${ }^{2}$ I am thankful to Juan Maldacena, Shiraz Minwalla and Suvrat Raju for pointing out that this index actually does not count chiral primaries as opposed to the claim which was made in the original version of this paper.
} 
Born-Oppenheimer approximation to count the chiral primaries. In this approximation method all chiral primaries actually correspond to gauge invariant chiral operators made out of the lowest components of the chiral superfields in the Lagrangian. Under the renormalization group flow some of the chiral primaries could disappear or new chiral primaries could appear. Supersymmetry, however, should somewhat protect the chiral primaries.

Typically, there are many relations in the chiral ring which can make the counting of chiral primaries quite involved. Using those methods one can compare the spectra of chiral primaries in superconformal field theories that are related by complicated dualities like Seiberg duality, which act in very non trivial ways on the field content.

The paper is organized as follows: In section 2 I use an $S U(2 \mid 1)$ subgroup of the $S U(2,2 \mid 1)$ superconformal algebra to derive the BPS bound for chiral primaries and prove the existence of an index. In section 3 I construct the Killing spinors on $S^{3} \times \mathbb{R}$ which are needed in section 4 to derive the field content and the Lagrangians of $\mathcal{N}=1$ supersymmetric field theories on this space. A similar approach has been used in [10] to construct supersymmetric gauge theories on curved manifolds. Furthermore I perform a twist on the field theory by replacing the Hamiltonian $H$ by $H-\frac{3}{2} J$, where $J$ is the R-charge. As a warmup exercise I count chiral primaries for some ungauged theories in section 5. To describe chiral primaries in a Born-Oppenheimer approximation I do a consistent truncation of the field theory to some supersymmetric quantum mechanics which has a mass term for the bosons only. The free theory turns out to be described by a harmonic oscillator in a frame which is rotating at the frequency of the oscillator. From this picture it is clear that there is an infinite degeneracy of zero energy states corresponding to an infinite number of chiral primaries. For Wess-Zumino models I use cohomology arguments to relate the chiral primaries to elements of the chiral ring. Even though it is believed that most Wess-Zumino models are not flowing to interacting fixed points this is a good warmup exercise for section 6 where I generalize this formalism to describe chiral primaries in gauge theories. For gauge theories this consistent truncation does not seem to work, but one can still use the chiral ring arguments. As an example for an application of this I count the chiral primaries for an $S U(2)$ SYM with 3 flavors and its Seiberg dual, which is just a Wess-Zumino model. The the results on both sides of the duality agrees, however a new chiral ring relation in the SYM is required to truncate the powers of the gaugino bilinears $S$ at first order 
already instead of the well known perturbative relation [1]

$$
S^{2}=0
$$

which truncates the powers of $S$ at second order.

This method could also be used to count BPS states that preserve 6 supersymmetries in theories with extended superconformal symmetry (see e.g. [6]). Or in theories with adjoint or bifundamental matter like [12, 13]. In those applications there exists a large $N$ limit and a gravity dual. One would then count BPS geometries.

\section{Group Theory}

\subsection{The algebra}

The $\mathcal{N}=1$ superconformal group in four dimensions is $S U(2,2 \mid 1)$, which contains $S U(2,2) \times U(1)_{R}$ as maximal bosonic subgroup. The supersymmetry generators $Q$ transform in the $4_{1}$ and their Hermitean conjugates $Q^{\dagger}$ in the $\overline{4}_{-1}$.

We want to put a four dimensional $\mathcal{N}=1$ superconformal field theory on $\mathbb{R} \times S^{3}$ which is the boundary of $A d S_{5}$ in global coordinates. The subgroup of the conformal group consisting of isometries of $\mathbb{R} \times S^{3}$ is $U(1) \times S O(4)$. The supersymmetry generators decompose as follows

$$
\begin{array}{ccc}
S U(2,2) \times U(1)_{R} & \rightarrow & S U(2)_{l} \times S U(2)_{r} \times U(1) \times U(1)_{R} \\
4_{1} & \rightarrow & (2,1)_{\frac{1}{2}, 1} \oplus(1,2)_{-\frac{1}{2}, 1} \\
\overline{4}_{-1} & \rightarrow & (2,1)_{-\frac{1}{2},-1} \oplus(1,2)_{\frac{1}{2},-1}
\end{array}
$$

Let $H, J_{i}$ and $\tilde{J}_{i}$ be the Hermitean generators of the isometry group $S U(2)_{l} \times$ $S U(2)_{r} \times U(1)$. The off diagonal blocks in $S U(2,2)$ are the conformal generators ${ }^{3} K_{\alpha \dot{\beta}}$

\footnotetext{
${ }^{3}$ The undotted lower case Greek indices refer to the $S U(2)_{l}$ and the dotted lower case Greek indices refer to the $S U(2)_{r}$.
} 
and $\left(K_{\alpha \dot{\beta}}\right)^{\dagger}$. In this basis the conformal algebra is given by

$$
\begin{aligned}
{[H, H] } & =0, \\
{\left[H, J_{i}\right] } & =0, \\
{\left[H, \tilde{J}_{i}\right] } & =0, \\
{\left[H, K_{\alpha \dot{\beta}}\right] } & =-K_{\alpha \dot{\beta}} \\
{\left[J_{i}, J_{j}\right] } & =i \epsilon_{i j k} J_{k}, \\
{\left[J_{i}, \tilde{J}_{j}\right] } & =0, \\
{\left[J_{i}, K_{\alpha \dot{\beta}}\right] } & =K_{\gamma \dot{\beta}} \sigma^{i \gamma}, \\
{\left[\tilde{J}_{i}, \tilde{J}_{j}\right] } & =i \epsilon_{i j k} \tilde{J}_{k}, \\
{\left[\tilde{J}_{i}, K_{\alpha \dot{\beta}}\right] } & =K_{\alpha \dot{\gamma}} \sigma^{i \dot{\alpha}}, \\
{\left[K_{\alpha \dot{\beta}}, K_{\gamma \dot{\delta}}\right] } & =0, \\
{\left[K_{\alpha \dot{\beta}},\left(K_{\gamma \dot{\gamma}}\right)^{\dagger}\right] } & =\delta_{\alpha}^{\gamma} \delta_{\dot{\beta}}^{\dot{\delta}} H-2 \delta_{\alpha}^{\gamma} \sigma_{(P)}^{i} \dot{\delta} \dot{\beta} \tilde{J}_{i}-2 \sigma_{(P)}^{i}{ }^{\gamma}{ }_{\alpha} \delta_{\dot{\beta}}^{\delta} J_{i}
\end{aligned}
$$

where $\sigma_{(P)}^{i}{ }_{\beta}^{\alpha}$ and $\sigma_{(P)}^{i}{ }_{\dot{\beta}}^{\dot{\beta}}$ are the Pauli matrices.

To construct the $\mathcal{N}=1$ superconformal algebra, one has to add the Hermitean generator $J$ of the $U(1)_{R}$ symmetry. $J$ commutes with all other bosonic generators. Finally the supersymmetry generators are $Q_{\alpha}$ which transform in the $(2,1)_{-\frac{1}{2},-1}$ and $S_{\dot{\alpha}}$ which transform in the $(1,2)_{-\frac{1}{2}, 1}$ together with their Hermitean conjugates $\left(Q_{\alpha}\right)^{\dagger}$ and $\left(S_{\dot{\alpha}}\right)^{\dagger}$. The bosonic generators have the following commutation relations with the supercharges:

$$
\begin{aligned}
{\left[H, Q_{\alpha}\right] } & =-\frac{1}{2} Q_{\alpha}, & {\left[H, S_{\dot{\alpha}}\right] } & =-\frac{1}{2} S_{\dot{\alpha}}, \\
{\left[J, Q_{\alpha}\right] } & =-Q_{\alpha}, & {\left[J, S_{\dot{\alpha}}\right] } & =S_{\dot{\alpha}}, \\
{\left[J_{i}, Q_{\alpha}\right] } & =Q_{\beta} \sigma_{(P)}^{i}{ }^{\beta}, & {\left[J_{i}, S_{\dot{\alpha}}\right] } & =0, \\
{\left[\tilde{J}_{i}, Q_{\alpha}\right] } & =0, & {\left[\tilde{J}_{i}, S_{\dot{\alpha}}\right] } & =S_{\dot{\beta}} \sigma_{(P)}^{i} \dot{\beta} \dot{\alpha} \\
{\left[K_{\alpha \dot{\beta}}, Q_{\gamma}\right] } & =0, & {\left[K_{\alpha \dot{\beta}}, S_{\dot{\gamma}}\right] } & =0, \\
{\left[\left(K_{\alpha \dot{\beta}}\right)^{\dagger}, Q_{\gamma}\right] } & =\delta_{\gamma}^{\alpha}\left(S_{\dot{\beta}}\right)^{\dagger}, & {\left[\left(K_{\alpha \dot{\beta}}\right)^{\dagger}, S_{\dot{\gamma}}\right] } & =\delta_{\dot{\gamma}}^{\dot{\beta}}\left(Q_{\alpha}\right)^{\dagger},
\end{aligned}
$$

The anti-commutation relations between the supercharges are:

$$
\begin{aligned}
\left\{Q_{\alpha}, Q_{\beta}\right\} & =0, \\
\left\{S_{\dot{\alpha}}, S_{\dot{\beta}}\right\} & =0 \\
\left\{Q_{\alpha},\left(Q_{\beta}\right)^{\dagger}\right\} & =\delta_{\alpha}^{\beta}\left(H-\frac{3}{2} J\right)-4 \sigma_{(P)}^{i}{ }_{\alpha} J_{i}, \\
\left\{S_{\dot{\alpha}},\left(S_{\dot{\beta}}\right)^{\dagger}\right\} & =\delta_{\dot{\alpha}}^{\dot{\beta}}\left(H+\frac{3}{2} J\right)-4 \sigma_{(P)}^{i} \dot{\beta} \dot{\alpha} \tilde{J}_{i}, \\
\left\{Q_{\alpha}, S_{\dot{\beta}}\right\} & =-2 K_{\alpha \dot{\beta}}, \\
\left\{Q_{\alpha},\left(S_{\dot{\beta}}\right)^{\dagger}\right\} & =0 .
\end{aligned}
$$


The coefficients in those anticommutation relations can be fixed by imposing the Jacobi identity and using (A.2).

\subsection{Chiral primaries}

There are important constraints from the unitatrity of representations of the superconformal group. We choose to label states by their quantum numbers under the isometry group together with the $U(1)_{R}$ symmetry. From the commutation relations of the

other generators with the Hamiltonian $H$ it follows that $K_{\alpha \dot{\beta}}, Q_{\alpha}$ and $S_{\dot{\beta}}$ are lowering operators, whereas their Hermitean conjugates are raising operators.

A primary operator is an operator that is annihilated by all the lowering operators. i.e. it is a state of lowest energy in a representation of the superconformal group. All other states in the same representation can be reached by applying raising operators and operators in the isometry group. A primary operator specifies a whole representation.

From the anti-commutation relations of the supercharges one can derive very important unitarity bounds

$$
-H \leq \frac{3}{2} J \leq H .
$$

Let us now concentrate on states $|\psi\rangle$ that saturate the right inequality. Those states are called chiral primary states. It is not hard to derive from the anti-commutation relations (2.4) that $|\psi\rangle$ satisfies

$$
Q_{\alpha}|\psi\rangle=\left(Q_{\alpha}\right)^{\dagger}|\psi\rangle=J_{i}|\psi\rangle=0 .
$$

Furthermore the state $S_{\dot{\alpha}}|\psi\rangle$ violates the unitarity bound and has to vanish. For this reason we get

$$
S_{\dot{\alpha}}|\psi\rangle=K_{\alpha \dot{\beta}}|\psi\rangle=0 .
$$

This implies that $|\psi\rangle$ is a primary operator which is also annihilated by the raising operators $\left(Q_{\alpha}\right)^{\dagger}$.

Finally it is not hard to derive a bound on the $\tilde{J}_{i}$ quantum numbers which implies that $|\psi\rangle$ can have maximally spin $H$ under the $S U(2)_{r}$. Since there are typically infinitely many chiral primaries in a superconformal field theory it is useful to count them weighted by their conformal weight

$$
Z_{\chi}(x)=\operatorname{tr}_{\chi} x^{H},
$$

where $x=e^{-\beta}$. 


\subsection{An index}

The superconformal algebra has a $\mathbb{Z}_{2}$ outer automorphism $(-1)^{F}$ which leaves all the bosonic symmetry generators invariant, negates all the supercharges and maps unitary representations to themselves. This suggests the existence of an index $\operatorname{tr}(-1)^{F}$. However, since the number of states is infinite, we need to introduce a regulator. In order for exact cancellations between Bosons and Fermions to happen, the regulator has to commute with some of the supercharges. There are two such regulators

$$
e^{-\frac{3 \beta}{2}\left(H-\frac{1}{2} J\right)} \quad \text { and } \quad e^{-\frac{3 \beta}{2}\left(H+\frac{1}{2} J\right)} .
$$

Because of the BPS bound it is easy to see that

$$
\frac{3}{2}\left(H \pm \frac{1}{2} J\right) \geq H
$$

which shows that the two regulators are good regulators for $\beta>0$.

Let us concentrate on the first regulator. It commutes with $H, J, J_{i}, \tilde{J}_{i}, Q_{\alpha}$ and $\left(Q_{\alpha}\right)^{\dagger}$. Those generate the subgroup $S U(2 \mid 1)_{l} \times S U(2)_{r} \times U(1) \in S U(2,2 \mid 1)$.

The $S U(2 \mid 1)_{l}$ is generated by $H^{\prime}=H-\frac{3}{2} J, J_{i}, Q_{\alpha}$ and $\left(Q_{\alpha}\right)^{\dagger}$. Representations can be split up into representations of $S U(2)_{l} \times U(1)_{h^{\prime}}$. Irreducible unitary representations can be labeled by the $S U(2)_{l} \times U(1)_{h^{\prime}}$ representation $\left(h^{\prime}, j_{l}\right)$ with the lowest $h^{\prime}$, they have to satisfy the BPS bound

$$
2 j_{l} \leq h^{\prime}
$$

There are three types of representations of $S U(2 \mid 1)_{l}$ (For a more general approach to representation theory of supergroups using Young tableaux see [14]):

- Long representations satisfy $2 j_{l}<h^{\prime}$. They contain three or four $S U(2) \times U(1)$ representations:

$$
\begin{gathered}
\left(h^{\prime}, 0\right) \oplus\left(h^{\prime}+1, \frac{1}{2}\right) \oplus\left(h^{\prime}+2,0\right) \\
\text { and } \\
\left(h^{\prime}, j_{l}\right) \oplus\left(h^{\prime}+1, j_{l}-\frac{1}{2}\right) \oplus\left(h^{\prime}+1, j_{l}+\frac{1}{2}\right) \oplus\left(h^{\prime}+2, j_{l}\right) .
\end{gathered}
$$

The index of a long representation vanishes.

- Short representations satisfy $2 j_{l}=h^{\prime}, h^{\prime} \geq 1$. They contain two $S U(2) \times U(1)$ representations:

$$
\left(h^{\prime}=2 j_{l}, j_{l}\right) \oplus\left(h^{\prime}+1=2 j_{l}+1, j_{l}-\frac{1}{2}\right) .
$$


The index of a short representation is \pm 1 . One can associate a semi-long multiplet of $S U(2,2 \mid 1)$ to those states [15], this corresponds to a semiconserved or a conserved superfield.

- The trivial representation satisfies $h^{\prime}=j_{l}=0$, the index is \pm 1 . As shown in the last section this corresponds to chiral representations of $S U(2,2 \mid 1)$.

For a short representation one gets $h-\frac{3}{2} j=2 j_{l}$. For this reason

$$
\operatorname{ind}\left(e^{-\beta}\right)=\operatorname{tr}(-1)^{F} e^{-\frac{3 \beta}{2}\left(H-\frac{1}{2} J\right)}
$$

counts the number of semi-short representations weighed by $h+j_{l}$ together with chiral primaries weighted by their conformal dimension. It is easy to see that inserting the other regulator into the trace would count short multiplets and anti-chiral primaries, where the shortening is due to a BPS bound involving $j_{r}$, the quantum number under $S U(2)_{r}$.

Finally, the $S U(2)_{r}$ generators commute with the supercharges $Q_{\alpha}$ and $\left(Q_{\alpha}\right)^{\dagger}$ and one can restrict the index to specific representations of $S U(2)_{r}{ }^{4}$ :

$$
\operatorname{ind}\left(e^{-\beta}, j_{r}\right)=\operatorname{tr}_{j_{r}}(-1)^{F} e^{-\frac{3 \beta}{2}\left(H-\frac{1}{2} J\right)}
$$

An implication of this for local conformal field theories is: The $S O(3)$ rotations on $S^{3}$ around a given fixed point are generated by the diagonal subgroup of $S U(2)_{l} \times S U(2)_{r}$. Since the chiral primaries are singlets under $S U(2)_{l}$ the $S U(2)_{r}$ quantum number is actually the spin of the chiral primary state. Using the spin statistics theorem one can argue that all chiral primaries contributing to the index have fermion number $(-1)^{2 j_{r}}$.

If the theory has more internal symmetries, the generators of those can be inserted in the trace as well.

To define the index actually only the $S U(2 \mid 1)$ subalgebra of the full conformal group was used ${ }^{5}$. For this reason the index can be defined for theories which are not conformal. This allows to compute the index in a non conformal field theory in the UV, which flows to a conformal fixed point in the IR.

The $S U(2 \mid 1)$ is very similar to the supertranslation algebra. Indeed the supertranslation algebra is a Inönü-Wigner contraction of $S U(2 \mid 1)$. The bosonic generators of

\footnotetext{
${ }^{4}$ This can be done by inserting the quadratic Casimir $\tilde{J}_{i} \tilde{J}_{i}$ of $S U(2)_{r}$ into the trace.

${ }^{5}$ Actually only the subalgebra generated by $H-\frac{3}{2} J-2 J_{3}, Q_{1}$ and $\left(Q_{1}\right)^{\dagger}$, i.e. $S U(1 \mid 1)$ is needed to define the index.
} 
$S U(2 \mid 1)$ correspond to translations of $S^{3} \times \mathbb{R}$ on which the theory is defined and the contraction is the large volume limit of the sphere. The index that is defined above is actually very similar to the usual Witten index [8] which is defined on $T^{3} \times \mathbb{R}$ and uses the supertranslation algebra as supersymmetry group. However, the Witten index is counting vacua of the theory in flat space as opposed to semi-short representations.

\section{$3 \quad$ Killing Spinors}

In order to calculate any of the quantities defined in the last section in an actual field theory we need to derive the classical Lagrangians for supersymmetric field theories on $S^{3} \times \mathbb{R}$. In a local field theory, the symmetries are generated by local currents. Space time symmetries are generated by vector fields, whereas internal symmetries are generated by scalars with values in the Lie algebra of the internal symmetry group. Supersymmetries are generated by Grassmann valued spinor fields, the Killing spinors.

Because of the appearance of anomalous dimensions it is in general impossible to construct classical Lagrangians on $\mathbb{R} \times S^{3}$ which preserve the whole superconformal symmetry. However one can construct classical Lagrangians on $\mathbb{R} \times S^{3}$ which preserve the isometry group, the $U(1)_{R}$ symmetry and half of the supersymmetries. For this purpose it is useful to introduce the anti-Hermitean operators

$$
\delta_{K^{0}}^{(H)}=i K^{0} H, \quad \delta_{K^{i}}^{\left(J_{i}\right)}=i K^{i} J_{i}, \quad \delta_{\tilde{K}^{i}}^{\left(\tilde{J}_{i}\right)}=i \tilde{K}^{i} \tilde{J}_{i}, \quad \text { and } \quad \delta_{\Sigma}^{(J)}=i \Sigma J
$$

which generate the preserved Bosonic symmetries and the anti-Hermitean operators

$$
\delta_{\hat{\zeta}}^{(Q)}=i \hat{\zeta}^{\alpha} Q_{\alpha}+i\left(\hat{\zeta}^{\alpha}\right)^{*}\left(Q_{\alpha}\right)^{\dagger},
$$

which generate the preserved supersymmetries. Note that $\hat{\zeta}^{\alpha}$ are Grassmann numbers.

The bosonic isometry algebra $U(1) \times S U(2)_{l} \times S U(2)_{r}$ can be realized by Killing vector fields on $\mathbb{R} \times S^{3}$

$$
K^{0} \partial_{t}, \quad K^{i} \sigma_{i}^{(L)} \quad \text { and } \quad \tilde{K}^{i} \sigma_{i}^{(R)},
$$

where the left and right derivatives are defined in appendix B. Those Killing vector fields act on the fields by through appropriately covariantized Lie derivatives. The $U(1)_{R}$ symmetry is realized by a constant real scalar field on $\mathbb{R} \times S^{3}$. It acts on the fields through multiplication weighed by an appropriate charge. 
In order to construct a supersymmetric field theory on $\mathbb{R} \times S^{3}$, let us first construct the Killing spinors $\zeta$ which generate the supersymmetries. The commutation relations between the bosonic symmetry generators and the supersymmetry generators

$$
\begin{aligned}
{\left[\delta_{K^{0}}^{(H)}, \delta_{\hat{\zeta}}^{(Q)}\right] } & =\delta_{-\frac{i K^{0}}{2} \hat{\zeta}}^{(Q)}, \\
{\left[\delta_{K^{i}}^{\left(J_{i}\right)}, \delta_{\hat{\zeta}}^{(Q)}\right] } & =\delta_{i K^{i} \sigma^{i} \hat{\zeta}}^{(Q)}, \\
{\left[\delta_{\tilde{K}^{i}}^{\left(\tilde{J}_{i}\right)}, \delta_{\hat{\zeta}}^{(Q)}\right] } & =0 \\
{\left[\delta_{\Sigma}^{(J)}, \delta_{\hat{\zeta}}^{(Q)}\right] } & =\delta_{-i \Sigma \hat{\zeta}}^{(Q)}
\end{aligned}
$$

imply a linear action of the bosonic symmetry generators on the Grassmann parameters $\hat{\zeta}$.

On the other hand the parameters $\hat{\zeta}$ should be mapped to the corresponding Killing spinors $\zeta$ by a linear map $M$. One can come up with a natural action of the bosonic symmetries on the Killing spinors. The requirement that the diagram

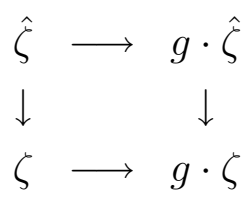

commutes implies the Killing spinor equations.

To deal with spinors on $\mathbb{R} \times S^{3}$ we set up a vielbein

$$
e^{0}=R_{1} d t \quad \text { and } \quad e^{i}=R_{2} \sigma_{(R)}^{i},
$$

the gamma matrix conventions are defined in appendix $\mathrm{A}$. The relative normalization of the two constants $R_{1}$ and $R_{2}$ cannot be fixed by the isometry algebra. It can be determined by conformal invariance or as we will see in the next section through the supersymmetry algebra.

In this frame the action of the bosonic symmetries on the Killing spinors can be realized by

$$
\begin{aligned}
R\left(\delta_{K^{0}}^{(H)}\right) \zeta & =K^{0} \partial_{t} \zeta \\
R\left(\delta_{K^{i}}^{\left(J_{i}\right)}\right) \zeta & =K^{i}\left(\sigma_{i}^{(L)}+\frac{1}{8} \epsilon_{i j k} \gamma^{j k}\left(\mathbb{1}+\gamma^{5}\right)\right) \zeta \\
R\left(\delta_{\tilde{K}^{i}}^{\left(\tilde{J}_{i}\right)}\right) \zeta & =\tilde{K}^{i}\left(\sigma_{i}^{(R)}+\frac{1}{8} \epsilon_{i j k} \gamma^{j k}\left(\mathbb{1}-\gamma^{5}\right)\right) \zeta \\
R\left(\delta_{\Sigma}^{(J)}\right) \zeta & =-i \Sigma \zeta .
\end{aligned}
$$


For $\delta_{K^{0}}^{(H)}$ and $\delta_{\tilde{K}^{i}}^{\left(\tilde{J}_{i}\right)}$ the commutativity of the diagram (3.8) implies the Killing spinor equations

$$
\begin{aligned}
\left(\partial_{t}+\frac{i}{2}\right) \zeta & =0, \\
\left(\sigma_{i}^{(R)}+\frac{1}{8} \epsilon_{i j k} \gamma^{j k}\left(\mathbb{1}-\gamma^{5}\right)\right) \zeta & =0 .
\end{aligned}
$$

Those equations are integrable and have a 4 dimensional solution space. In order to find an isomorphism to the Grassmann parameters $\hat{\zeta}$, we need to restrict the Killing spinors to have positive chirality

$$
\gamma^{5} \zeta=\zeta
$$

which implies that

$$
\sigma_{i}^{(R)} \zeta=\sigma_{i}^{(L)} \zeta=0
$$

The commutativity of the diagram (3.8) for $\delta_{\Sigma}^{(J)}$ transformations is automatically satisfied. The action of $\delta_{K^{i}}^{\left(J_{i}\right)}$ on Killing spinors is actually given by

$$
R\left(\delta_{K^{i}}^{\left(J_{i}\right)}\right) \zeta=\frac{K^{i}}{4} \epsilon_{i j k} \gamma^{j k} \zeta
$$

The commutativity of the diagram (3.8) then implies that the isomorphism $M$ is given by

$$
\zeta=M \cdot \hat{\zeta}=e^{-\frac{i}{2} t}\left(\begin{array}{l}
\hat{\zeta} \\
0
\end{array}\right) .
$$

This result can also be derived by restricting the Killing spinors on $A d S_{5}$ in global coordinates to the boundary as is done in [16].

\section{Construction of the Field Theory}

The first step in constructing a supersymmetric field theory is to find an action of the Killing spinors on fields, such that the anti-commutation relations between the supercharges are satisfied. The relevant anti-commutation relation for realizing the supersymmetry transformations is

$$
\left[\delta_{\hat{\zeta}_{1}}^{(Q)}, \delta_{\hat{\zeta}_{2}}^{(Q)}\right]=-2 \delta_{\operatorname{Im}\left(\hat{\zeta}_{1}^{\dagger} \hat{\zeta}_{2}\right)}^{(H)}+3 \delta_{\operatorname{Im}\left(\hat{\zeta}_{1}^{\dagger} \hat{\zeta}_{2}\right)}^{(J)}+8 \delta_{\operatorname{Im}\left(\hat{\zeta}_{1}^{\dagger} \sigma_{(P)}^{i} \hat{\zeta}_{2}\right)}^{\left(J_{i}\right)}
$$

Because the closure of the supersymmetry algebra contains internal symmetries it is hard to use a superspace approach like in [17] to this problem instead I will use a component field approach. 


\subsection{Chiral multiplets and the Wess-Zumino model}

Like in flat space, a chiral superfield consists of a complex scalar $\phi$, a chiral fermion $\psi$ and an auxiliary complex scalar $F$. They can be put together as a vector $(\phi, \psi, F)$. This chiral multiplet has to transform as a linear representation of the symmetry algebra. For the beginning we assume that the chiral multiplet is not gauged. Then it transforms under the Bosonic symmetries as

$$
\begin{aligned}
\delta_{K^{0}}^{(H)}(\phi, \psi, F) & =\left(K^{0} \partial_{t} \phi, K^{0} \partial_{t} \psi, K^{0} \partial_{t} F\right), \\
\delta_{K^{i}}^{\left(J_{i}\right)}(\phi, \psi, F) & =\left(K^{i} \sigma_{i}^{(L)} \phi, K^{i}\left(\sigma_{i}^{(L)}+\frac{1}{4} \epsilon_{i j k} \gamma^{j k}\right) \psi, K^{i} \sigma_{i}^{(L)} F\right), \\
\delta_{\tilde{K}^{i}}^{\left(\tilde{J}_{i}\right)}(\phi, \psi, F) & =\left(\tilde{K}^{i} \sigma_{i}^{(R)} \phi, \tilde{K}^{i} \sigma_{i}^{(R)} \psi, \tilde{K}^{i} \sigma_{i}^{(R)} F\right), \\
\delta_{\Sigma}^{(J)}(\phi, \psi, F) & =(-i q \Sigma \phi,-i(q-1) \Sigma \psi,-i(q-2) \Sigma F) .
\end{aligned}
$$

In the presence of many internal $U(1)$ symmetries the conformal R-charge q of a chiral multiplet at the conformal fixed point can be determined using a-maximization [18]. The supersymmetry transformation has the form

$$
\begin{aligned}
& \delta_{\tilde{\zeta}}^{(Q)}(\phi, \psi, F)= \\
& \left(\tilde{\zeta} \psi,\left(\partial_{t}+\frac{3 i q}{2}\right) \phi \gamma^{0} \zeta^{\odot}-2 \sigma_{i}^{(L)} \phi \gamma^{i} \zeta^{\odot}+\zeta F,-i \bar{\zeta} \gamma^{0}\left(\partial_{t}+i \frac{3 q-5}{2}\right) \psi+2 i \bar{\zeta} \gamma^{i} \sigma_{i}^{(L)} \psi\right) .
\end{aligned}
$$

Using identities from appendix $\mathrm{A}$ one can show that those transformations close according to (4.1).

It is not hard to verify that the Lagrangians

$$
\begin{aligned}
\mathcal{L}_{0}= & \left(\partial_{t}-i \frac{3 q-2}{2}\right) \phi^{*}\left(\partial_{t}+i \frac{3 i q-2}{2}\right) \phi-4 \sigma_{i}^{(L)} \phi^{*} \sigma_{i}^{(L)} \phi-\phi^{*} \phi \\
& +i \bar{\psi} \gamma^{0}\left(\partial_{t}+i \frac{3 q-2}{2}\right) \psi-2 i \bar{\psi} \gamma^{i}\left(\sigma_{i}^{(L)}+\frac{1}{8} \epsilon_{i j k} \gamma^{j k}\right) \psi+F^{*} F, \\
\mathcal{L}_{W}= & W^{\prime}(\phi) F-\frac{1}{2} W^{\prime \prime}(\phi) \tilde{\psi} \psi+\text { h.c. }
\end{aligned}
$$

with

$$
W^{\prime}(\phi)=c \phi^{-\frac{q-2}{q}}
$$

are supersymmetric. As expected the superpotential $W(\phi)$ has to have R-charge 2 . The kinetic terms in $\mathcal{L}_{0}$ fixes the relative normalization of the two vielbein coefficients $R_{1}$ and $R_{2}$ to be

$$
R_{1}=-2 R_{2}=R .
$$




\section{$4.2 \quad$ Vector multiplets}

A vector multiplet contains a gauge field $\mathcal{A}_{\mu}$ a chiral fermion $\lambda$, the gaugino, and a real auxiliary field $D$ all with values in the Lie algebra of the gauge group ${ }^{6}$. The gaugino and the auxiliary field transform in the adjoint representation of the gauge group. Let the gauge field be

$$
\mathcal{A}=\mathcal{A}_{0} d t+\mathcal{A}_{i} \sigma_{(R)}^{i}
$$

Then the gauge field strength is

$$
\begin{aligned}
\mathcal{F}= & d \mathcal{A}+\mathcal{A} \wedge \mathcal{A} \\
= & d t \wedge \sigma_{(R)}^{i}\left(\partial_{t} \mathcal{A}_{i}-\sigma_{i}^{(L)} \mathcal{A}_{0}+\mathcal{A}_{0} \mathcal{A}_{i}-\mathcal{A}_{i} \mathcal{A}_{0}\right) \\
& +\frac{1}{2} \sigma_{(R)}^{i} \wedge \sigma_{(R)}^{j}\left(\sigma_{i}^{(L)} \mathcal{A}_{j}-\sigma_{j}^{(L)} \mathcal{A}_{i}+\epsilon_{i j k} \mathcal{A}_{k}+\mathcal{A}_{i} \mathcal{A}_{j}-\mathcal{A}_{j} \mathcal{A}_{i}\right) .
\end{aligned}
$$

This allows to read off the components

$$
\begin{aligned}
\mathcal{F}_{0 i} & =\frac{1}{2}\left(\partial_{t} \mathcal{A}_{i}-\sigma_{i}^{(L)} \mathcal{A}_{0}+\mathcal{A}_{0} \mathcal{A}_{i}-\mathcal{A}_{i} \mathcal{A}_{0}\right) \\
\mathcal{F}_{i j} & =\frac{1}{2}\left(\sigma_{i}^{(L)} \mathcal{A}_{j}-\sigma_{j}^{(L)} \mathcal{A}_{i}+\epsilon_{i j k} \mathcal{A}_{k}+\mathcal{A}_{i} \mathcal{A}_{j}-\mathcal{A}_{j} \mathcal{A}_{i}\right) .
\end{aligned}
$$

The appropriate gauge covariant derivatives are

$$
\begin{aligned}
\mathcal{D} \mathcal{F} & =d \mathcal{F}+\mathcal{A} \wedge \mathcal{F}-\mathcal{F} \wedge \mathcal{A} \\
\mathcal{D}_{0} \lambda & =\partial_{t} \lambda+\mathcal{A}_{0} \lambda-\lambda \mathcal{A}_{0} \\
\mathcal{D}_{i} \lambda & =\sigma_{i}^{(L)} \lambda+\mathcal{A}_{i} \lambda-\lambda \mathcal{A}_{i}, \\
\mathcal{D} D & =d D+\mathcal{A} D-D \mathcal{A} .
\end{aligned}
$$

The Bianchi identity is

$$
\mathcal{D} \mathcal{F}=0,
$$

which implies

$$
\mathcal{D}_{i} \mathcal{F}_{j 0}+\mathcal{D}_{j} \mathcal{F}_{0 i}+\mathcal{D}_{0} \mathcal{F}_{i j}=\epsilon_{i j k} \mathcal{F}_{0 k} \quad \text { and } \quad \epsilon_{i j k} \mathcal{D}_{i} \mathcal{F}_{j k}=0
$$

Gauge transformations are generated by a Lie algebra valued generator $\Lambda$ as follows

$$
\begin{aligned}
\delta_{\Lambda}^{(g)} \mathcal{A} & =d \Lambda+\mathcal{A} \Lambda-\Lambda \mathcal{A} \\
\delta_{\Lambda}^{(g)} \mathcal{F} & =\mathcal{F} \Lambda-\Lambda \mathcal{F} \\
\delta_{\Lambda}^{(g)} \lambda & =\lambda \Lambda-\Lambda \lambda \\
\delta_{\Lambda}^{(g)} D & =D \Lambda-\Lambda D .
\end{aligned}
$$

\footnotetext{
${ }^{6} \mathrm{I}$ am using anti Hermitean Lie algebra generators.
} 
The vector multiplet $(\mathcal{A}, \lambda, D)$ has to transform in a gauge covariant way under the bosonic symmetries. Looking at the expressions for the components of the gauge field strength the natural transformations are

$$
\begin{aligned}
\delta_{K^{0}}^{(H)}\left(\mathcal{A}_{0}, \mathcal{A}_{i}, \lambda, D\right) & =\left(0,2 K^{0} \mathcal{F}_{0 i}, K^{0} \mathcal{D}_{0} \lambda, K^{0} \mathcal{D}_{0} D\right) \\
\delta_{K^{i}}^{\left(J_{i}\right)}\left(\mathcal{A}_{0}, \mathcal{A}_{i}, \lambda, D\right) & =\left(2 K^{j} \mathcal{F}_{j 0}, 2 K^{j} \mathcal{F}_{j i}, K^{j}\left(\mathcal{D}_{j}+\frac{1}{4} \epsilon_{j k l} \gamma^{k l}\right) \lambda, K^{i} \mathcal{D}_{i} D\right) \\
\delta_{\Sigma}^{(J)}\left(\mathcal{A}_{0}, \mathcal{A}_{i}, \lambda, D\right) & =(0,0,-i \Sigma \lambda, 0)
\end{aligned}
$$

Note that it is harder to write down the gauge covariant transformation law under $\delta_{\tilde{K}^{i}}^{\left(\tilde{J}_{i}\right)}$ in the frame we chose. Since we won't need this transformation law, we will not write it down. The supersymmetry transformations

$$
\begin{aligned}
\delta_{\hat{\zeta}}^{(Q)} \mathcal{A}_{0} & =2 \operatorname{Re}\left(\bar{\zeta} \gamma_{0} \lambda\right) \\
\delta_{\hat{\zeta}}^{(Q)} \mathcal{A}_{i} & =-\operatorname{Re}\left(\bar{\zeta} \gamma_{i} \lambda\right) \\
\delta_{\hat{\zeta}}^{(Q)} \mathcal{F}_{0 i} & =\mathcal{D}_{i} \operatorname{Re}\left(\bar{\lambda} \gamma^{0} \zeta\right)-\frac{1}{2} \mathcal{D}_{0} \operatorname{Re}\left(\bar{\lambda} \gamma^{i} \zeta\right), \\
\delta_{\hat{\zeta}}^{(Q)} \mathcal{F}_{i j} & =-\frac{1}{2} \mathcal{D}_{i} \operatorname{Re}\left(\bar{\lambda} \gamma^{j} \zeta\right)+\frac{1}{2} \mathcal{D}_{j} \operatorname{Re}\left(\bar{\lambda} \gamma^{i} \zeta\right)-\frac{1}{2} \epsilon_{i j k} \operatorname{Re}\left(\bar{\lambda} \gamma^{k} \zeta\right), \\
\delta_{\hat{\zeta}}^{(Q)} \lambda & =-2 i \mathcal{F}_{0 i} \gamma^{0 i} \zeta+2 i \mathcal{F}_{i j} \gamma^{i j} \zeta+D \zeta \\
\delta_{\hat{\zeta}}^{(Q)} \lambda^{\odot} & =2 i \mathcal{F}_{0 i} \gamma^{0 i} \zeta^{\odot}-2 i \mathcal{F}_{i j} \gamma^{i j} \zeta^{\odot}+D \zeta^{\odot}, \\
\delta_{\hat{\zeta}}^{(Q)} D & =\operatorname{Im}\left(\bar{\zeta} \gamma^{0}\left(\mathcal{D}_{0}-\frac{3 i}{2}\right) \lambda\right)-2 \operatorname{Im}\left(\bar{\zeta} \gamma^{i} \mathcal{D}_{i} \lambda\right)
\end{aligned}
$$

close according to (4.1). A supersymmetric Lagrangian is

$$
\mathcal{L}_{g}=\frac{1}{g^{2}}\left(4 \operatorname{tr} \mathcal{F}_{0 i} \mathcal{F}_{0 i}-8 \operatorname{tr} \mathcal{F}_{i j} \mathcal{F}_{i j}+i \operatorname{tr} \bar{\lambda} \gamma^{0} \mathcal{D}_{0} \lambda-2 i \operatorname{tr} \bar{\lambda} \gamma^{i}\left(\mathcal{D}_{i}+\frac{1}{8} \epsilon_{i j k} \gamma^{j k}\right) \lambda-\operatorname{tr} D^{2}\right)
$$

This Lagrangian the covariantized flat-space Lagrangian. The $\theta$-term

$$
\mathcal{L}_{\theta}=\theta \epsilon_{i j k} \mathcal{F}_{0 i} \mathcal{F}_{j k}
$$

is separately supersymmetric. For an Abelian gauge group there is a Fayet-Ilioupoulous term

$$
\mathcal{L}_{F I}=\kappa \operatorname{tr}\left(D-\mathcal{A}_{0}\right) .
$$

Note that the integral of this Fayet-Ilioupoulous term is actually gauge invariant. However, this Fayet-Ilioupoulous term modifies the Gauss law constraint drastically by producing a background charge which can lead to difficulties for the quantum theory. 


\subsection{Gauge invariant interactions}

Finally we want to couple chiral multiplets to gauge fields. In order to do so we need to replace all derivatives by covariant ones and introduce a few additional terms to cancel unwanted contributions. Gauge transformations act on a chiral multiplet in the representation $\rho$ of the gauge group in the usual way

$$
\delta_{\Lambda}^{(g)}(\phi, \psi, F)=\left(-\Lambda^{(\rho)} \phi,-\Lambda^{(\rho)} \psi,-\Lambda^{(\rho)} F\right)
$$

The action of the Bosonic symmetry is easily generalized

$$
\begin{aligned}
\delta_{K^{0}}^{(H)}(\phi, \psi, F) & =\left(K^{0} \mathcal{D}_{0} \phi, K^{0} \mathcal{D}_{0} \psi, K^{0} \mathcal{D}_{0} F\right), \\
\delta_{K^{i}}^{\left(J_{i}\right)}(\phi, \psi, F) & =\left(K^{i} \mathcal{D}_{i} \phi, K^{i}\left(\mathcal{D}_{i}+\frac{1}{4} \epsilon_{i j k} \gamma^{j k}\right) \psi, K^{i} \mathcal{D}_{i} F\right), \\
\delta_{\Sigma}^{(J)}(\phi, \psi, F) & =(-i q \Sigma \phi,-i(q-1) \Sigma \psi,-i(q-2) \Sigma F),
\end{aligned}
$$

where the gauge covariant derivatives are defined as follows

$$
\mathcal{D}_{0} \phi=\partial_{t} \phi+\mathcal{A}_{0}^{(\rho)} \phi \quad \text { and } \quad \mathcal{D}_{i} \phi=\sigma_{i}^{(L)} \phi+\mathcal{A}_{i}^{(\rho)} \phi \quad \text { etc. }
$$

The supersymmetry transformations then have the form

$$
\begin{aligned}
\delta_{\zeta}^{(Q)} \phi & =\tilde{\zeta} \psi \\
\delta_{\zeta}^{(Q)} \psi & =\left(\mathcal{D}_{0}+\frac{3 i q}{2}\right) \phi \gamma^{0} \zeta^{\odot}-2 \mathcal{D}_{i} \phi \gamma^{i} \zeta^{\odot}+\zeta F \\
\delta_{\zeta}^{(Q)} F & =-i \bar{\zeta} \gamma^{0}\left(\mathcal{D}_{0}+i \frac{3 q-5}{2}\right) \psi+2 i \bar{\zeta} \gamma^{i} \mathcal{D}_{i} \psi-2\left(\bar{\zeta}\left(\lambda^{\odot}\right)^{(\rho)}\right) \phi
\end{aligned}
$$

and close according to (4.1).

The supersymmetric matter Lagrangian is

$$
\begin{aligned}
\mathcal{L}_{0}= & \left(\mathcal{D}_{0}-i \frac{3 q-2}{2}\right) \phi^{\dagger}\left(\mathcal{D}_{0}+i \frac{3 i q-2}{2}\right) \phi-4 \mathcal{D}_{i} \phi^{\dagger} \mathcal{D}_{i} \phi-\phi^{\dagger} \phi \\
& +i \bar{\psi} \gamma^{0}\left(\mathcal{D}_{0}+i \frac{3 q-2}{2}\right) \psi-2 i \bar{\psi} \gamma^{i}\left(\mathcal{D}_{i}+\frac{1}{8} \epsilon_{i j k} \gamma^{j k}\right) \psi+F^{\dagger} F \\
& +2 i \phi^{\dagger} D^{(\rho)} \phi-2 i \phi^{\dagger} \tilde{\lambda}(\rho) \psi+2 \bar{\psi}\left(\lambda^{\odot}\right)^{(\rho)} \phi, \\
\mathcal{L}_{W}= & W^{\prime}(\phi) F-\frac{1}{2} W^{\prime \prime}(\phi) \tilde{\psi} \psi+\text { h.c. },
\end{aligned}
$$

where the superpotential $W$ is gauge invariant and has R-charge 2.

\subsection{A twist}

Since the chiral primaries saturate the BPS bound

$$
H-\frac{3}{2} J \geq 0
$$


they are the ground states of a twisted theory, where the original Hamiltonian $H$ is replaced by $H^{\prime}=H-\frac{3}{2} J$. In that twisted theory they can be treated using the Born-Oppenheimer approximation. The twist is done by simply doing the replacement

$$
\begin{gathered}
\lambda=e^{-\frac{3 i}{2} t} \lambda^{\prime} \\
\phi=e^{-\frac{3 i q}{2} t} \phi^{\prime}, \quad \psi=e^{-\frac{3 i(q-1)}{2} t} \psi^{\prime} \text { and } F=e^{-\frac{3 i(q-2)}{2} t} F^{\prime}
\end{gathered}
$$

in all expressions. From now on I will only deal with the twisted theory and omit the primes. The Lagrangians then have the form

$$
\begin{aligned}
\mathcal{L}_{g}= & \frac{1}{g^{2}}\left(4 \operatorname{tr} \mathcal{F}_{0 i} \mathcal{F}_{0 i}-8 \operatorname{tr} \mathcal{F}_{i j} \mathcal{F}_{i j}\right) \\
& +\frac{1}{g^{2}}\left(i \operatorname{tr} \bar{\lambda} \gamma^{0} \mathcal{D}_{0} \lambda-2 i \operatorname{tr} \bar{\lambda} \gamma^{i}\left(\mathcal{D}_{i}+\frac{1}{4} \epsilon_{i j k} \gamma^{j k}\right) \lambda-\operatorname{tr} D^{2}\right) \\
\mathcal{L}_{\theta}= & \theta \epsilon_{i j k} \mathcal{F}_{0 i} \mathcal{F}_{j k} \\
\mathcal{L}_{F I}= & \kappa \operatorname{tr}\left(D-\mathcal{A}_{0}\right) \\
\mathcal{L}_{0}= & \left(\mathcal{D}_{0}+i\right) \phi^{\dagger}\left(\mathcal{D}_{0}-i\right) \phi-4 \mathcal{D}_{i} \phi^{\dagger} \mathcal{D}_{i} \phi-\phi^{\dagger} \phi \\
& +i \bar{\psi} \gamma^{0}\left(\mathcal{D}_{0}-i\right) \psi-2 i \bar{\psi} \gamma^{i} \mathcal{D}_{i} \psi+F^{\dagger} F \\
& +2 i \phi^{\dagger} D^{(\rho)} \phi-2 i \phi^{\dagger} \tilde{\lambda}(\rho) \psi+2 \bar{\psi}\left(\lambda^{\odot}\right)^{(\rho)} \phi \\
\mathcal{L}_{W}= & W^{\prime}(\phi) F-\frac{1}{2} W^{\prime \prime}(\phi) \tilde{\psi} \psi
\end{aligned}
$$

Note that the spatial derivatives of the fermions are gauge covariant, but have a different connection from the metric connection. This turns out to be more natural in the twisted theory, since it emphasizes the lowest energy harmonics.

This twisted theory has a supersymmetry algebra of the form

$$
\left[\delta_{\hat{\zeta}_{1}}^{(Q)}, \delta_{\hat{\zeta}_{2}}^{(Q)}\right]=-2 \delta_{\operatorname{Im}\left(\hat{\zeta}_{1}^{\dagger} \hat{\zeta}_{2}\right)}^{(H)}+8 \delta_{\operatorname{Im}\left(\hat{\zeta}_{1}^{\dagger} \sigma_{(P)}^{i} \hat{\zeta}_{2}\right)}^{\left(J_{i}\right)}
$$

with the time translations just being the appropriate gauge covariant time derivative. The $U(1)_{R}$ symmetry does not have to be enforced anymore. This allows for inhomogeneous superpotentials. The twisted Lagrangians could be derived by superfield methods with the superspace being the $S U(2 \mid 1)$ group manifold.

Those Lagrangians look like Lagrangians of matter coupled to a background $U(1)$ gauge field. However, this $U(1)$ is typically broken by the superpotential.

In order to calculate the index defined in section 2.3 it is actually more convenient to twist the theory even more by defining the new Hamiltonian $H^{\prime \prime}=H-\frac{3}{2} J-2 J_{3}$. In this 
twisted theory the index can be calculated using the Born-Oppenheimer approximation 9. Using $H^{\prime \prime}$ as a Hamiltonian amounts to going to a rotating coordinate system on $S^{3} \times \mathbb{R}$.

\section{$5 \quad$ Ungauged theories}

This section is devoted to the theory of a free chiral multiplet and the Wess Zumino model. Usually, the Wess Zumino model is not really physical since it is believed to be IR free, but it is still interesting to demonstrate techniques that lead to the chiral ring. Furthermore, the Seiberg dual of $S U(2)$ SYM with 3 flavors is a Wess Zumino model with 15 chiral multiplets. I will count the chiral primaries of that theory in section 6.2.

\subsection{Reduction to quantum mechanics}

Chiral primaries are zero energy eigenstates of the Hamiltonian $H^{\prime}=H-\frac{3}{2} J$. For this reason it is useful to reduce the field theory on $S^{3} \times \mathbb{R}$ to a supersymmetric quantum mechanics. This is done by restricting to the lowest energy modes of the theory.

In a weak coupling limit the potential energy for the chiral bosons is

$$
\sigma_{i}^{(L)} \phi^{\dagger} \sigma_{i}^{(L)} \phi+\phi^{\dagger} \phi
$$

This is minimized for the S-wave mode of $\phi$. The fermion on the other hand minimizes the potential energy for the $S U(2)_{L}$ doublet mode

$$
\sigma_{i}^{(L)} \psi=0 .
$$

With these restrictions the Lagrangian reduces to

$$
\begin{aligned}
L_{0} & =\left(\partial_{t}+i\right) \phi^{\dagger}\left(\partial_{t}-i\right) \phi-\phi^{\dagger} \phi+i \bar{\psi}_{0} \gamma^{0}\left(\partial_{t}-i\right) \psi_{0}+F^{\dagger} F, \\
L_{W} & =W^{\prime}(\phi) F-\frac{1}{2} W^{\prime \prime}(\phi) \tilde{\psi}_{0} \psi_{0} .
\end{aligned}
$$

The supercharges of the twisted field theory are doublets under the $S U(2)_{L}$. For this reason the reduced theory, has to include $S U(2)_{L}$ singlet fields together with their superpartners which are $S U(2)_{L}$ doublets. The $S U(2)_{L}$ is now an internal symmetry of the Lagrangian and the supersymmetry algebra still closes as in equation (4.59). The supersymmetry acts on the fields as

$$
\begin{aligned}
\delta_{\zeta}^{(Q)} \phi & =\tilde{\zeta} \psi \\
\delta_{\zeta}^{(Q)} \psi & =\partial_{t} \phi \gamma^{0} \zeta^{\odot}+\zeta F, \\
\delta_{\zeta}^{(Q)} F & =-i \bar{\zeta} \gamma^{0}\left(\partial_{t}-i\right) \psi
\end{aligned}
$$


The time translation is just the derivative $\partial_{t}$ and the $S U(2)_{L}$ rotation acts on $\psi$ as

$$
\delta_{K^{i}}^{\left(J_{i}\right)} \psi=\frac{1}{4} \epsilon_{i j k} \gamma^{j k} \psi
$$

Note that this mechanics is some mechanics in a rotating frame. It is actually the mechanics that can be gotten from dimensional reduction of a $\mathcal{N}=1, d=4$ theory to 1 dimension and adding a mass term for the chiral bosons. The supersymmetry is generated by a time dependent Killing spinor. For this reason the supersymmetry relates states of different energies. A similar supersymmetric quantum mechanics was found in [19].

When doing such a reduction, one might be missing fermionic zero modes and associated anomalies. In the twisted theory all fields are coupled to a constant background $U(1)$ gauge field in time direction. All fermions in chiral multiplets have charge $\frac{1}{2}$ and the gauginos have charge $-\frac{3}{2}$ under that $U(1)$. Since this background $U(1)$ gauge field is flat and the Pontrjagin class of the curvature of $S^{3} \times \mathbb{R}$ is vanishing, there are no net fermionic zero modes and the reduction is valid.

\subsection{The canonical formalism}

The canonical momenta conjugate to $\phi, \psi$ are

$$
p=\left(\partial_{t}+i\right) \phi^{\dagger}, \quad p^{\dagger}=\left(\partial_{t}-i\right), \quad \pi=-i \psi^{\dagger}
$$

and the canonical (anti-) commutation relations are

$$
[\phi, p]=-i, \quad\left[\phi^{\dagger}, p^{\dagger}\right]=-i, \quad\left\{\psi_{\alpha},\left(\psi_{\beta}\right)^{\dagger}\right\}=\delta_{\alpha}^{\beta}
$$

with all other (anti-) commutators vanishing. The unusual sign in the canonical commutation relations is due to the unitarity requirement of the fermionic sector combined with supersymmetry.

The Lagrangian then gets Legendre transformed to the Hamiltonian

$$
\begin{aligned}
H= & \left(p-i \phi^{\dagger}\right)\left(p^{\dagger}+i \phi\right)+\psi^{\dagger} \psi \\
& +\left|W^{\prime}(\phi)\right|^{2}-W^{\prime \prime}(\phi) \psi_{1} \psi_{2}+\left(W^{\prime \prime}(\phi)\right)^{\dagger} \psi_{1}^{\dagger} \psi_{2}^{\dagger}
\end{aligned}
$$

The $U(1)_{R}$ symmetry generator is

$$
J=-i q p \phi+i q \phi^{\dagger} p^{\dagger}-\frac{1}{2}(q-1)\left(2 \psi_{0}^{\dagger} \psi_{0}-\mathbb{1}\right),
$$


the $S U(2)_{L}$ generators are

$$
J_{i}=-\psi^{\dagger} \sigma_{(P)}^{i} \psi
$$

the supersymmetry generators ${ }^{7}$ are

$$
Q_{\alpha}=-\left(p-i \phi^{\dagger}\right) \psi_{\alpha}+i\left(W^{\prime}(\phi)\right)^{\dagger} \epsilon_{\alpha \beta} \psi_{\beta}^{\dagger}
$$

and the Fermion number operator is

$$
(-1)^{F}=(-1)^{\psi^{\dagger} \psi}
$$

\subsection{The free theory}

Let us first consider the theory of a free chiral multiplet. The Hamiltonian is given by

$$
H_{0}=\left(p-i \phi^{\dagger}\right)\left(p^{\dagger}+i \phi\right)+\psi^{\dagger} \psi
$$

this can be analyzed using the creation and annihilation operators

$$
\begin{aligned}
& a_{1}^{\dagger}=\frac{1}{\sqrt{2}}\left(p^{\dagger}-i \phi\right), \quad a_{1}=\frac{1}{\sqrt{2}}\left(p+i \phi^{\dagger}\right), \quad\left[a_{1}, a_{1}^{\dagger}\right]=1, \\
& a_{2}^{\dagger}=\frac{1}{\sqrt{2}}\left(p-i \phi^{\dagger}\right), \quad a_{2}=\frac{1}{\sqrt{2}}\left(p^{\dagger}+i \phi\right), \quad\left[a_{2}, a_{2}^{\dagger}\right]=1, \\
& b_{1}^{\dagger}=\psi_{1}^{\dagger}, \quad b_{1}=\psi_{1}, \quad\left\{b_{1}, b_{1}^{\dagger}\right\}=1 \text {, } \\
& b_{2}^{\dagger}=\psi_{2}^{\dagger}, \quad b_{2}=\psi_{2}, \quad\left\{b_{2}, b_{2}^{\dagger}\right\}=1 \text {, }
\end{aligned}
$$

with all other (anti-) commutation relations vanishing. Those operators define a Fock space with a vacuum

$$
a_{1}|0\rangle=a_{2}|0\rangle=b_{1}|0\rangle=b_{2}|0\rangle=0
$$

and states

$$
\left|n_{1}, n_{2}, \eta_{1}, \eta_{2}\right\rangle=\frac{1}{\sqrt{n_{1} ! n_{2} !}}\left(a_{1}^{\dagger}\right)^{n_{1}}\left(a_{2}^{\dagger}\right)^{n_{2}}\left(b_{1}^{\dagger}\right)^{\eta_{1}}\left(b_{2}^{\dagger}\right)^{\eta_{2}}|0\rangle .
$$

The Hamiltonian can be written as

$$
H_{0}=2 a_{2}^{\dagger} a_{2}+b_{1}^{\dagger} b_{1}+b_{2}^{\dagger} b_{2}
$$

and the $U(1)_{R}$ generator can be written as

$$
J=\frac{2}{3}\left(a_{1}^{\dagger} a_{1}-a_{2}^{\dagger} a_{2}\right)-\frac{1}{3}\left(b_{1}^{\dagger} b_{1}+b_{2}^{\dagger} b_{2}\right) .
$$

\footnotetext{
${ }^{7}$ Note that those generators are almost the generators of usual supersymmetric quantum mechanics, where the free part of the supercharge $-p \psi_{\alpha}$ is replaced by $-\left(p-i \phi^{\dagger}\right) \psi_{\alpha}$.
} 
The Hamiltonian obviously has the ground states $\left|n_{1}\right\rangle_{0}=\left|n_{1}, 0,-,-\right\rangle$

$$
H_{0}\left|n_{1}\right\rangle_{0}=0 \quad \text { and } \quad J\left|n_{1}\right\rangle_{0}=\frac{2 n_{1}}{3}\left|n_{1}\right\rangle_{0} .
$$

The Fermion number operator is given by

$$
(-1)^{F}=\left(1-2 b_{1}^{\dagger} b_{1}\right)\left(1-2 b_{2}^{\dagger} b_{2}\right)
$$

which implies that all the ground states are bosons. The chiral primaries are then counted by the partition function

$$
Z_{\chi}(x)=\sum_{m=0}^{\infty} x^{m}=\frac{1}{1-x}
$$

The above reasoning can be brought into a more familiar form by expressing the chiral primary states in terms of chiral operators $\phi$

$$
\left|n_{1}\right\rangle_{0}=\frac{1}{\sqrt{n_{1} !}}(-\sqrt{2} i \phi)^{n_{1}}|0\rangle .
$$

Then the chiral primary states are just polynomials in $\phi$ as expected.

\subsection{The Wess-Zumino model and the chiral ring}

We have seen in section 4.4 that the superpotential of the twisted theory can be deformed to an inhomogeneous one. The classical BPS equations then imply

$$
\partial_{t} \phi=0 \quad \text { and } \quad W^{\prime}(\phi)=0 .
$$

For this reason the classical theory has $n$ distinct supersymmetric vacua with a mass gap. This indicates that the theory has $n$ chiral primaries, all of the same fermion number similar as in $\left[8\right.$. However, it is impossible to see the $U(1)_{R}$ charges of those vacua with this method. To determine those, let us stay with the homogeneous superpotential and find solutions to the quantum BPS equations. This will also make a very natural connection with the chiral ring.

Chiral primaries satisfy

$$
J_{i}|\Omega\rangle=0 .
$$

Using $J_{3}$ implies that any chiral primary is a linear combination of states of the form $\left|m_{1}, m_{2},-,-\right\rangle$ and $\left|m_{1}, m_{2},+,+\right\rangle$ in the Fock space, they are $S U(2)_{l}$ singlets. Let us 
actually split the Hilbert space into a subspace $\mathcal{H}^{(+)}$of states which are $S U(2)_{l}$ singlets and a subspace $\mathcal{H}^{(-)}$of states which are $S U(2)_{l}$ doublets

$$
\mathcal{H}=\mathcal{H}^{(+)} \oplus \mathcal{H}^{(-)}
$$

The supersymmetry generators $\left(Q_{\alpha}\right)^{\dagger}$ are nilpotent operators of grade one in the above grading. The kernel and the image of $\left(Q_{1}\right)^{\dagger}$ and $\left(Q_{2}\right)^{\dagger}$ inside $\mathcal{H}^{(+)}$agree. Furthermore

$$
H=\left\{Q_{1},\left(Q_{1}\right)^{\dagger}\right\}
$$

inside $\mathcal{H}^{(+)}$. For this reason the zero modes of the Hamiltonian are harmonic representatives of the cohomology of $\left(Q_{1}\right)^{\dagger}$ inside $\mathcal{H}^{(+)}$. This means that in order to count chiral primaries it is enough to count $S U(2)_{l}$ invariant cohomology classes of $\left(Q_{1}\right)^{\dagger}$.

The supercharge $\left(Q_{1}\right)^{\dagger}$ can be written as

$$
\left(Q_{1}\right)^{\dagger}=-\sqrt{2} a_{2} b_{1}^{\dagger}-i W^{\prime}\left(\frac{a_{2}-a_{1}^{\dagger}}{\sqrt{2} i}\right) b_{2} .
$$

It is clear that the states $|m, 0,-,-\rangle$ are $\left(Q_{1}\right)^{\dagger}$-closed, using the result of the last section those states can be written as

$$
\phi^{m}|0\rangle
$$

Also, it is clear that states of the form

$$
W^{\prime}(\phi) \phi^{m}|0\rangle
$$

are $\left(Q_{1}\right)^{\dagger}$-exact. For this reason the cohomology of $\left(Q_{1}\right)^{\dagger}$ inside $\mathcal{H}^{(+)}$is described by polynomials in $\phi$ modulo $W^{\prime}(\phi)$, which is the chiral ring, as expected.

Note that the states $\phi^{m}|0\rangle$ are in general not the harmonic representatives of the cohomology classes, i.e. they are not the chiral primaries. In order to prove that this is all of the cohomology, one can note that number of chiral primaries gotten in this way agrees with the classical argument. One can also use spectral sequence arguments like in [20].

All the chiral primaries have the fermion number 0 and are counted by

$$
Z_{\chi}(x)=\sum_{m=0}^{n-1} x^{\frac{3 m}{n+1}}=\frac{1-x^{\frac{3 n}{n+1}}}{1-x^{\frac{3}{n+1}}} .
$$

The chiral primaries are all generated by $\phi$, which has quantum numbers $(1,1)$ under $S U(2)_{L} \times S U(2)_{R}$. For this reason all the chiral primaries are singlets under the $S U(2)_{R}$. In order to get chiral primaries of different spins, we will need to go to gauge theories. 


\section{Gauge theories and the chiral ring}

The supersymmetric quantum mechanics of the last section was actually not gotten by a proper Kaluza Klein reduction, but by consistent truncation. Since the supercharge does not commute with the Hamiltonian, one always has to keep massive superpartners of massless fields in order to get a supersymmetric theory. For gauge theories there does not seem to be a consistent truncation to quantum mechanics. However, we will use some of the technology of the last section and the chiral ring to find chiral primaries. The chiral ring that we are deriving here is similar to the chiral ring in flat space [21, 11, 22], however it does not agree with it. For example the multi fermion operators derived in [23] are not present in the chiral ring on $S^{3} \times \mathbb{R}$. In flat space the chiral ring is used to describe supersymmetric vacua 24] similarly on $S^{3} \times \mathbb{R}$ the chiral ring can be used to describe chiral primaries ${ }^{8}$.

\subsection{The chiral Ring}

In the last section we used the following two properties of chiral primaries: They are invariant under the $S U(2)_{L}$ and they are elements of the $Q_{1}^{\dagger}$ cohomology. We will make use of those properties to deal with gauge theories.

All $S U(2)_{L}$ invariant $Q_{1}^{\dagger}$ closed states are also $Q_{2}^{\dagger}$ closed. Furthermore any $S U(2)_{L}$ invariant $Q_{1}^{\dagger}$ exact state is also $Q_{2}^{\dagger}$ exact. From this we conclude that the $S U(2)_{L}$ invariant subsector of the $Q_{1}^{\dagger}$ cohomology and the $Q_{2}^{\dagger}$ cohomology agree. For this reason we can restrict ourselves to the $Q_{1}^{\dagger}$ cohomology $^{9} H^{\bullet}\left(Q_{1}^{\dagger}\right)$.

In the $S U(2)_{L}$ invariant sector of the Hilbert space the Hamiltonian can actually be written as

$$
H=\left\{Q_{1}, Q_{1}^{\dagger}\right\}
$$

and zero energy eigenstates of the Hamiltonian again are in one to one correspondence with the $S U(2)_{L}$ invariant sector of the $Q_{1}^{\dagger}$ cohomology $H^{0}\left(Q_{1}^{\dagger}\right)$ : For every cohomology representative $|\Omega\rangle_{0} \in H^{0}\left(Q_{1}^{\dagger}\right), H|\Omega\rangle_{0}$ is $Q_{1}^{\dagger}$ exact, there is a harmonic representative $|\Omega\rangle_{0} \in H^{0}\left(Q_{1}^{\dagger}\right)$ in the cohomology class of $|\Omega\rangle_{0}$ and every state that is annihilated by $H$ is $Q_{1}^{\dagger}$ closed. This means that the zero energy eigenstates of $H$ can be represented by elements of $H^{0}\left(Q_{1}^{\dagger}\right)$.

\footnotetext{
${ }^{8}$ The number of elements in the chiral ring of flat space has been counted in 25.

${ }^{9}$ This is actually a better situation than for the chiral ring in Minkowski space.
} 
The full $Q_{1}^{\dagger}$ cohomology $H^{\bullet}\left(Q_{1}^{\dagger}\right)$ actually describes the short representations of $S U(2 \mid 1)$ and the index is the Euler character of the full cohomology [9].

The discussion above also shows the the elements of $H^{0}\left(Q_{1}^{\dagger}\right)$ are invariant under space time translations, just as elements of the chiral ring of theories in flat space are.

In order to construct $H^{0}\left(Q_{1}^{\dagger}\right)$ it is useful to use the Born-Oppenheimer approximation. In a weakly coupled theory the zeroth order approximation to the zero energy eigenstates of $H$ can be constructed by restricting the field theory to the massless modes of the fields. The gauge field on $S^{3} \times \mathbb{R}$ has no massless mode, neither have the fermions $\psi$. The only massless modes are the $(1,2)$ harmonic of the gaugino $\lambda_{\alpha}$ and the $(1,1)$ harmonic of the scalar $\phi$ and their conjugates. We will denote those lowest harmonics of $\lambda_{\alpha}$ and $\phi$ by $\lambda_{\alpha}$ and $\phi$. It is easy to see that those satisfy

$$
\left\{Q_{1}^{\dagger}, \lambda_{\alpha}\right\}=0 \quad \text { and } \quad\left[Q_{1}^{\dagger}, \phi\right]=0
$$

even for non vanishing coupling, whereas their conjugates are not $Q_{1}^{\dagger}$ closed. For this reason the elements of $H^{0}\left(Q_{1}^{\dagger}\right)$ can be represented by gauge invariant expressions made out of $\lambda_{\alpha}$ and $\phi$ acting on the vacuum modulo relations which show that some of those states are $Q_{1}^{\dagger}$ exact. Note that those cohomology representatives are in general not the harmonic ones for interacting theories.

This construction agrees with the operator state correspondence of conformal field theories. This is that chiral primaries can be described by gauge invariant polynomials in $\lambda_{\alpha}$ and $\phi$ acting on the unique conformal invariant vacuum.

The canonical commutation relations together with translation invariance imply the relations

$$
\left\{\lambda_{\alpha}, \lambda_{\beta}\right\}=0, \quad\left[\lambda_{\alpha}, \phi\right]=0 \quad \text { and } \quad[\phi, \phi]=0
$$

in the chiral ring. Furthermore, by varying the elementary fields and their canonical conjugates, one also gets the relations

$$
F^{\dagger}=W^{\prime}(\phi)=0 \quad \text { and } \quad \lambda_{\alpha}^{(R)} \phi^{(R)}=0
$$

in the chiral ring ${ }^{10}$, where in the second equation the gaugino is acting on a matter field in a given representation $R$. There might be further perturbative relations in

\footnotetext{
${ }^{10}$ The first relation is actually a classical relation and typically gets modified. In a regime where matter fields are integrated out it is replaced by the generalized Konishi anomaly [1], 22.
} 
the chiral ring, which are more model specific. Those perturbative relations might get nonperturbative corrections.

In the weak coupling nonperturbative corrections are due to instanton effects. On Euclidean $S^{3} \times S^{1}$ one can indeed construct instanton configurations. The index is the partition function on $S^{3} \times S^{1}$ with periodic boundary conditions on the fermions and no other operator insertions, for this reason it shouldn't receive any nonperturbative corrections. The partition function for chiral primaries, however has a projection operator on chiral primaries inserted and might receive instanton corrections.

This can be formulated in a different way. The only thing that matters about chiral ring relations for counting chiral primaries, is where the chiral ring gets truncated. This should not be affected by deformations of the relations due to nonperturbative effects. For example in a pure $\mathcal{N}=1$ super Yang Mills theory in flat space there is the perturbative chiral ring relation 11

$$
S^{N_{c}}=0
$$

where $S=\operatorname{tr} \lambda_{1} \lambda_{2}$ is the gaugino bilinear. This gets modified by nonperturbative effects to

$$
S^{N_{c}}=\Lambda^{3 N_{c}}
$$

In both cases the relation truncates the chiral ring at the same point.

On the other hand, the chiral primaries behave similar to vacua in flat space. There are cases know in flat space where vacua are destabilized by nonperturbative effects 26]. There the chiral ring constraints have no solution. Here, however we assume the existence of a conformally invariant vacuum and we create chiral primaries by acting with the harmonic representatives of the chiral ring.

The example in the next section seems to suggest that our method of counting chiral primaries works fairly well.

All the arguments presented here only depend on the symmetry group $S U(2 \mid 1)$ and not on the full superconformal group. For this reason it might be interesting to extend this analysis to non-conformal theories on $S^{3} \times \mathbb{R}$.

\subsection{Seiberg duality for $S U(2)$ super Yang Mills theory with 3 flavors}

For a $S U(2)$ super Yang Mills theory with 3 flavors we can actually count the chiral primaries. The fundamental and the antifundamental representations are the same 
which means that there are 6 fundamental matter fields $q$ all with $U(1)_{R}$ charge $\frac{2}{3}$. Using the properties of group characters (see appendix A of [27])

$$
n_{\text {singlet }}=\int_{G}[d g] \prod_{i} \chi_{R_{i}}(g)
$$

and

$$
\sum_{n=0}^{\infty} x^{\frac{n}{2}} \chi_{s y m^{n}(R)}(g)=\exp \left(\sum_{l=1}^{\infty} \frac{x^{\frac{l}{2}}}{l} \chi_{R}\left(g^{l}\right)\right)
$$

we can count the chiral primaries made of just matter fields. Their number is given by the matrix integral

$$
Z_{\chi, M}(x)=\int_{S U(2)}[d g] \exp \left(6 \sum_{l=1}^{\infty} \frac{x^{\frac{l}{2}}}{l} \chi_{f}\left(g^{l}\right)\right)^{6}
$$

Going to an eigenvalue basis for this matrix integral introduces a measure factor

$$
Z_{\chi, M}(x)=\frac{2}{\pi} \int_{\varphi=0}^{\pi} \sin ^{2} \varphi d \varphi \exp \left(12 \sum_{l=1}^{\infty} \frac{x^{\frac{l}{2}}}{l} \cos (l \varphi)\right)^{6}=\frac{2}{\pi} \int_{\varphi=0}^{\pi} \frac{\sin ^{2} \varphi d \varphi}{(1-2 \sqrt{x} \cos \varphi+x)^{6}}
$$

This integral can be solved

$$
Z_{\chi, M}(x)=\frac{1+6 x+6 x^{2}+x^{3}}{(1-x)^{9}} .
$$

The Seiberg dual [28, 23] has a trivial, gauge group. This means that it is a Wess Zumino model with dual 'meson' matter fields $M^{i j}$ in the antisymmetric representation of the $S U(6)$ flavor group. The superpotential is

$$
W=\epsilon_{i_{1} \cdots i_{6}} M^{i_{1} i_{2}} M^{i_{3} i_{4}} M^{i_{5} i_{6}}
$$

This imposes the chiral ring relations

$$
\epsilon_{i_{1} \cdots i_{6}} M^{i_{3} i_{4}} M^{i_{5} i_{6}}=0 .
$$

The dual 'meson' matter fields can be represented in terms of Young tableaux as

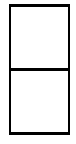


The $n$-th excited level is described by the symmetric product of the dual 'meson' matter fields. The superpotential constraint is that every term in the expansion of the symmetric product in young tableaux containing 4 or more boxes in one column is actually vanishing. Furthermore the symmetric product implies that the height of each column in a young tableaux is a multiple of 2. For this reason the only young tableaux contributing is

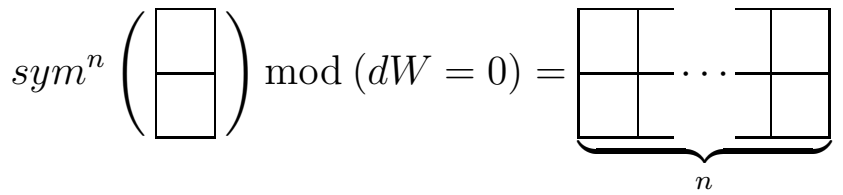

This representation has dimension

$$
d(n)=\frac{(n+5) !(n+4) !}{5 ! 4 !(n+1) ! n !}
$$

and the chiral primaries are counted by

$$
Z_{\chi}(x)=\sum_{n=0}^{\infty} d(n) x^{n}=\frac{1+6 x+6 x^{2}+x^{3}}{(1-x)^{9}} .
$$

This shows that the number of chiral primaries is preserved under Seiberg duality as long as on the gauge theory side of the duality only matter fields are taken into account.

Because of the relation (6.4) the gauginos on the gauge theory side can only contribute in terms of gaugino bilinears (see [29]). Furthermore there is the perturbative chiral ring relation

$$
S^{2}=0 .
$$

This is not good enough yet. However, the R-charges and flavor symmetry allow for an extra chiral ring relation of the form

$$
S \sim \operatorname{Pf}(q \cdot q) .
$$

Perturbatively the right hand side is vanishing since the matrix $q \cdot q$ has only rank 2 . Nonperturbatively this relation can make sense. As of now I was not able to prove such a relation, but it seems to be important for Seiberg duality to work.

\subsection{Seiberg duality in the conformal window}

In the conformal window $\frac{3 N_{c}}{2} \leq N_{f} \leq 3 N_{c}$ Seiberg duality [28] is the statement that two different gauge theories in the UV, an 'electric' theory and a 'magnetic' theory 
flow in the IR to the same fixed point. The 'electric' theory is a $S U\left(N_{c}\right)$ SYM with $N_{f}$ flavors $Q_{i}$ and $\tilde{Q}^{i}$, whereas the 'magnetic' theory is a $S U\left(N_{f}-N_{c}\right)$ SYM with $N_{f}$ flavors $q_{i}$ and $\tilde{q}^{i}$ and $N_{f}^{2}$ singlet fields $M_{i}{ }^{j}$ with a superpotential

$$
W=\tilde{q}^{i} M_{i}^{j} q_{j}
$$

For such theories it seems to be much harder to count chiral primaries. In the UV is fairly clear which gauge invariant expressions in terms of chiral operators are supposed to be mapped to each other under Seiberg duality. This map was made much more profound in the full gauge theory in 21] for matter fields and for the gaugino bilinear.

It is however less clear which chiral ring relations map to each other under Seiberg duality. Especially, when the gauginos are included, there appears a similar problem as in the previous section: On the electric side of the duality, there is the perturbative relation

$$
S_{e}^{N_{c}}=0,
$$

whereas on the magnetic side there is a similar relation

$$
S_{m}^{N_{f}-N_{c}}=0
$$

Furthermore the electric gaugino bilinear $S_{e}$ and the magnetic gaugino bilinear $S_{m}$ are proportional to each other. In order for the chiral rings on both sides of the duality to truncate at the same point, there have to be relations of the form

$$
S_{e}^{N_{f}-N_{c}} \sim \operatorname{det}(\tilde{q} q) \quad \text { and } \quad S_{m}^{N_{c}} \sim \operatorname{det}(\tilde{Q} Q)
$$

or something similar. Again the right hand sides are vanishing perturbatively, but might be nonvanishing by nonperturbative effects.

\section{Acknowledgments}

Research at the Perimeter Institute is supported in part by funds from NSERC of Canada.

I would like to thank the Benasque Center for physics, the CERN theory division and the Duke Center for Geometry and Theoretical Physics where part of the work was done.

I would like to thank Sujay Ashok, Paul Aspinwall, Itzhak Bars, David Berenstein, Alex Buchel, Freddy Cachazo, Rich Corrado, Mike Douglas, Laurent Freidel, Jaume 
Gomis, Nick Halmagyi, Ken Intriligator, Juan Maldacena, Shiraz Minwalla, Ronen Plesser, Maxim Pospelov, Suvrat Raju, Nati Seiberg, Nemani Suryanarayana and Nick Warner for useful discussions.

\section{A Some conventions}

In this appendix I set up some conventions for spinors and list some useful relations which are needed to prove the closure of the supersymmetry algebra and the invariance of Lagrangians under supersymmetry transformations. Pauli matrices are given by

$$
\sigma_{(P)}^{1}=\frac{1}{2}\left(\begin{array}{cc}
0 & 1 \\
1 & 0
\end{array}\right), \quad \sigma_{(P)}^{2}=\frac{1}{2}\left(\begin{array}{cc}
0 & -i \\
i & 0
\end{array}\right), \quad \sigma_{(P)}^{3}=\frac{1}{2}\left(\begin{array}{cc}
1 & 0 \\
0 & -1
\end{array}\right) .
$$

and satisfy the identity

$$
\delta_{\beta}^{\alpha} \delta_{\delta}^{\gamma}=\frac{1}{2} \delta_{\delta}^{\alpha} \delta_{\beta}^{\gamma}+2 \sum_{i} \sigma_{(P)}^{i}{ }^{\alpha}{ }_{\delta} \sigma_{(P)}^{i}{ }^{\gamma} \beta .
$$

In the $(-+++)$ metric convention the gamma matrices can be chosen as

$$
\begin{gathered}
\gamma^{0}=\left(\begin{array}{cc}
0 & -\mathbb{1} \\
\mathbb{1} & 0
\end{array}\right), \quad \gamma^{i}=2\left(\begin{array}{cc}
0 & \sigma^{i} \\
\sigma^{i} & 0
\end{array}\right) . \\
\gamma^{5}=i \gamma^{0123}=\left(\begin{array}{cc}
\mathbb{1} & 0 \\
0 & -\mathbb{1}
\end{array}\right)
\end{gathered}
$$

In those conventions the Hermitean conjugation is given by

$$
\left(\gamma^{\mu}\right)^{\dagger}=-C \gamma^{\mu} C^{-1} \quad \text { with } \quad C=\gamma^{0} \quad \text { and } \quad \bar{\zeta}=\zeta^{\dagger} C,
$$

the transpose is given by

$$
\left(\gamma^{\mu}\right)^{t}=D \gamma^{\mu} D^{-1} \quad \text { with } \quad D=\gamma^{135} \quad \text { and } \quad \tilde{\zeta}=\zeta^{t} D
$$

and the complex conjugate is given by

$$
\left(\gamma^{\mu}\right)^{*}=-B \gamma^{\mu} B^{-1} \quad \text { with } \quad B=\gamma^{2} \quad \text { and } \quad \zeta^{\odot}=B^{-1} \zeta^{*} .
$$


Chiral spinors satisfy the relations

$$
\begin{aligned}
\gamma^{5} \zeta & =\zeta \\
\bar{\zeta}_{1} \gamma^{0} \zeta_{2} & =-\hat{\zeta}_{1}^{\dagger} \hat{\zeta}_{2} \\
\bar{\zeta}_{1} \gamma^{i} \zeta_{2} & =-2 \hat{\zeta}_{1}^{\dagger} \sigma_{(P)}^{i} \hat{\zeta}_{2} \\
\tilde{\zeta}_{2} \gamma^{\mu} \zeta_{1}^{\odot} & =i \bar{\zeta}_{1} \gamma^{\mu} \zeta_{2} \\
\tilde{\zeta}_{1} \zeta_{2} & =\tilde{\zeta}_{2} \zeta_{1} \\
\epsilon_{i j k} \gamma^{j k} \psi & =-2 i \gamma^{0 i} \psi
\end{aligned}
$$

and the Fierz identities

$$
\begin{aligned}
\zeta_{1}^{\odot}\left(\tilde{\zeta}_{2} \psi\right) & =-\frac{i}{2}\left(\bar{\zeta}_{1} \gamma_{\nu} \zeta_{2}\right) \gamma^{\nu} \psi \\
\zeta_{1}\left(\bar{\zeta}_{2} \gamma^{\mu} \psi\right) & =-\frac{1}{2}\left(\bar{\zeta}_{2} \gamma_{\nu} \zeta_{1}\right) \gamma^{\nu} \gamma^{\mu} \psi \\
\zeta_{1}\left(\tilde{\zeta}_{2} \psi\right) & =-\frac{1}{2}\left(\tilde{\zeta}_{2} \zeta_{1}\right) \psi-\frac{1}{2}\left(\tilde{\zeta}_{2} \gamma^{0 i} \zeta_{1}\right) \gamma^{0 i} \psi
\end{aligned}
$$

\section{B Invariant forms and Killing vectors on $S U(2)$}

The left invariant 1-forms on $S U(2)$ are given by

$$
\begin{aligned}
\sigma_{(L)}^{1} & =\cos \varphi_{3} d \varphi_{1}+\sin \varphi_{3} \sin \varphi_{1} d \varphi_{2} \\
\sigma_{(L)}^{2} & =\sin \varphi_{3} d \varphi_{1}-\cos \varphi_{3} \sin \varphi_{1} d \varphi_{2}, \\
\sigma_{(L)}^{3} & =\cos \varphi_{1} d \varphi_{2}+d \varphi_{3}
\end{aligned}
$$

they satisfy

$$
d \sigma_{(L)}^{i}=\frac{1}{2} \epsilon_{i j k} \sigma_{(L)}^{j} \wedge \sigma_{(L)}^{k}
$$

The dual vectors are the Killing vectors for right multiplication

$$
\begin{aligned}
\sigma_{1}^{(R)} & =\cos \varphi_{3} \partial_{\varphi_{1}}+\frac{\sin \varphi_{3}}{\sin \varphi_{1}} \partial_{\varphi_{2}}-\sin \varphi_{3} \cot \varphi_{1} \partial_{\varphi_{3}}, \\
\sigma_{2}^{(R)} & =\sin \varphi_{3} \partial_{\varphi_{1}}-\frac{\cos \varphi_{3}}{\sin \varphi_{1}} \partial_{\varphi_{2}}+\cos \varphi_{3} \cot \varphi_{1} \partial_{\varphi_{3}}, \\
\sigma_{3}^{(R)} & =\partial_{\varphi_{3}},
\end{aligned}
$$

they satisfy

$$
\left[\sigma_{i}^{(R)}, \sigma_{j}^{(R)}\right]=-\epsilon_{i j k} \sigma_{k}^{(R)} \quad \text { and } \quad £_{\sigma_{i}^{(R)}} \sigma_{(L)}^{j}=-\epsilon_{i j k} \sigma_{(L)}^{k}
$$


Similarly, the right invariant 1-forms are

$$
\begin{aligned}
\sigma_{(R)}^{1} & =\cos \varphi_{2} d \varphi_{1}+\sin \varphi_{2} \sin \varphi_{1} d \varphi_{3}, \\
\sigma_{(R)}^{2} & =\sin \varphi_{2} d \varphi_{1}-\cos \varphi_{2} \sin \varphi_{1} d \varphi_{3}, \\
\sigma_{(R)}^{3} & =\cos \varphi_{1} d \varphi_{3}+d \varphi_{2}
\end{aligned}
$$

and the dual vectors to those are the Killing vectors for left multiplication

$$
\begin{aligned}
\sigma_{1}^{(L)} & =\cos \varphi_{2} \partial_{\varphi_{1}}+\frac{\sin \varphi_{2}}{\sin \varphi_{1}} \partial_{\varphi_{3}}-\sin \varphi_{2} \cot \varphi_{1} \partial_{\varphi_{2}}, \\
\sigma_{2}^{(L)} & =\sin \varphi_{2} \partial_{\varphi_{1}}-\frac{\cos \varphi_{2}}{\sin \varphi_{1}} \partial_{\varphi_{3}}+\cos \varphi_{2} \cot \varphi_{1} \partial_{\varphi_{2}}, \\
\sigma_{3}^{(L)} & =\partial_{\varphi_{2}},
\end{aligned}
$$

they satisfy

$$
\begin{aligned}
d \sigma_{(L)}^{i} & =\frac{1}{2} \epsilon_{i j k} \sigma_{(L)}^{j} \wedge \sigma_{(L)}^{k}, \\
{\left[\sigma_{i}^{(R)}, \sigma_{j}^{(R)}\right] } & =-\epsilon_{i j k} \sigma_{k}^{(R)} \\
£_{\sigma_{i}^{(L)}}^{(L)} \sigma_{(R)}^{j} & =-\epsilon_{i j k} \sigma_{(R)}^{k}
\end{aligned}
$$

and

$$
\begin{aligned}
{\left[\sigma_{i}^{(L)}, \sigma_{j}^{(R)}\right] } & =0, \\
£_{\sigma_{i}^{(L)}} \sigma_{(L)}^{j} & =0 \\
£_{\sigma_{i}^{(R)}} \sigma_{(R)}^{j} & =0 .
\end{aligned}
$$




\section{References}

[1] D. Berenstein, "A toy model for the ads/cft correspondence," JHEP 07 (2004) 018, hep-th/0403110.

[2] H. Lin, O. Lunin, and J. Maldacena, "Bubbling ads space and 1/2 bps geometries," JHEP 10 (2004) 025, hep-th/0409174.

[3] D. Berenstein, "Large n bps states and emergent quantum gravity," hep-th/0507203.

[4] R. Gopakumar, "From free fields to ads," Phys. Rev. D70 (2004) 025009, hep-th/0308184.

[5] R. Dijkgraaf and C. Vafa, "Matrix models, topological strings, and supersymmetric gauge theories," Nucl. Phys. B644 (2002) 3-20, hep-th/0206255.

[6] H. Lin and J. Maldacena, "Fivebranes from gauge theory," hep-th/0509235.

[7] C. Römelsberger, "An index to count chiral primaries in n=1 d=4 scft's," String Theory Seminar at Duke (September 15, 2005).

[8] E. Witten, "Constraints on supersymmetry breaking," Nucl. Phys. B202 (1982) 253.

[9] C. Römelsberger, "work in progress,".

[10] M. Blau, "Killing spinors and sym on curved spaces," JHEP 11 (2000) 023, hep-th/0005098.

[11] F. Cachazo, M. R. Douglas, N. Seiberg, and E. Witten, "Chiral rings and anomalies in supersymmetric gauge theory," JHEP 12 (2002) 071, hep-th/0211170.

[12] R. G. Leigh and M. J. Strassler, "Exactly marginal operators and duality in four-dimensional n=1 supersymmetric gauge theory," Nucl. Phys. B447 (1995) 95-136, hep-th/9503121.

[13] I. R. Klebanov and E. Witten, "Superconformal field theory on threebranes at a calabi-yau singularity," Nucl. Phys. B536 (1998) 199-218, hep-th/9807080. 
[14] A. Baha Balantekin and I. Bars, "Representations of supergroups," J. Math. Phys. 22 (1981) 1810.

[15] S. Ferrara and A. Zaffaroni, "Superconformal field theories, multiplet shortening, and the $\operatorname{ads}(5) / \operatorname{scft}(4)$ correspondence," hep-th/9908163.

[16] K. Okuyama, "N = 4 sym on r x s(3) and pp-wave," JHEP 11 (2002) 043, hep-th/0207067.

[17] J. Wess and J. Bagger, "Supersymmetry and supergravity," . Princeton, USA: Univ. Pr. (1992) 259 p.

[18] K. Intriligator and B. Wecht, "The exact superconformal r-symmetry maximizes a," Nucl. Phys. B667 (2003) 183-200, hep-th/0304128.

[19] N.-w. Kim, T. Klose, and J. Plefka, "Plane-wave matrix theory from $\mathrm{n}=4$ super yang-mills on r x s**3," Nucl. Phys. B671 (2003) 359-382, hep-th/0306054.

[20] S. Kachru and E. Witten, "Computing the complete massless spectrum of a landau- ginzburg orbifold," Nucl. Phys. B407 (1993) 637-666, hep-th/9307038.

[21] D. Kutasov, A. Schwimmer, and N. Seiberg, "Chiral rings, singularity theory and electric-magnetic duality," Nucl. Phys. B459 (1996) 455-496, hep-th/9510222.

[22] A. Brandhuber, H. Ita, H. Nieder, Y. Oz, and C. Römelsberger, "Chiral rings, superpotentials and the vacuum structure of $\mathrm{n}=1$ supersymmetric gauge theories," Adv. Theor. Math. Phys. 7 (2003) 269-305, hep-th/0303001.

[23] C. Beasley and E. Witten, "New instanton effects in supersymmetric qcd," JHEP 01 (2005) 056, hep-th/0409149.

[24] P. Svrcek, "Chiral rings, vacua and gaugino condensation of supersymmetric gauge theories," JHEP 08 (2004) 036, hep-th/0308037.

[25] M. R. Douglas, "The statistics of string / m theory vacua," JHEP 05 (2003) 046, hep-th/0303194.

[26] I. Affleck, M. Dine, and N. Seiberg, "Supersymmetry breaking by instantons," Phys. Rev. Lett. 51 (1983) 1026. 
[27] O. Aharony, J. Marsano, S. Minwalla, K. Papadodimas, and M. Van Raamsdonk, "The hagedorn / deconfinement phase transition in weakly coupled large n gauge theories," Adv. Theor. Math. Phys. 8 (2004) 603-696, hep-th/0310285.

[28] N. Seiberg, "Electric - magnetic duality in supersymmetric nonabelian gauge theories," Nucl. Phys. B435 (1995) 129-146, hep-th/9411149.

[29] N. Seiberg, "Adding fundamental matter to 'chiral rings and anomalies in supersymmetric gauge theory'," JHEP 01 (2003) 061, hep-th/0212225. 